\title{
Efectos de Activación e Inhibición en el Crecimiento del Coleoptilo y del Sistema Radicular de Semillas de Trigo Provocados por Radiación Láser Ultravioleta
}

\author{
Mauricio Hernández y Alexandre Michtchenko \\ (1) Sección de Estudios de Posgrado e Investigación (SEPI-ESIME-Zacatenco). \\ Unidad Profesional Adolfo López Mateos, Zacatenco, Del. Gustavo A. Madero, C.P. 07738, \\ México D.F. - México (e-mail: mahernandezvi@ipn.mx; amichtchenko@ipn.mx).
}

Recibido Jun. 23, 2011; Aceptado Ago. 08, 2011; Versión Final recibida Ago. 27, 2011

\section{Resumen}

Se ha investigado los efectos de la radiación láser infrarroja sobre el crecimiento del coleoptilo y del sistema radicular en semillas de trigo de la variedad Náhuatl (Triticum aestivum L). Se usó un láser de diodo con longitud de onda $\lambda=980 \mathrm{~nm}$ y se realizaron quince tratamientos con diferentes densidades de potencia y tiempos de exposición. Cada tratamiento láser se aplicó una vez antes de la siembra en experimentos elaborados bajo condiciones de laboratorio. El análisis estadístico mostró activaciones e inhibiciones importantes en el crecimiento del coleoptilo y del sistema radicular. Los resultados muestran que pese a que el coleoptilo sufrió modificaciones en su crecimiento el sistema radicular fue el más susceptible a la radiación láser, mostrando una activación máxima de $15.2 \%$ y una inhibición máxima de $17 \%$.

Palabras clave: laser de diodo, radiación láser infrarroja, trigo, coleoptilo, sistema radicular.

\section{Effects of Activation and Inhibition on the Growth of the Coleoptile and on the Root System of Wheat Seeds caused by Infrared Laser Radiation}

\begin{abstract}
In this study the effects of infrared laser radiation on the growth of coleoptile and root system in wheat seeds Nahuatl variety (Triticum aestivum $L$ ) were investigated. A laser diode with wavelength $\lambda=980 \mathrm{~nm}$ was used and fifteen laser treatments were performed with different power densities and exposure times. Each laser treatment was applied once before sowing in experiments carried on under laboratory conditions. The statistical analysis showed important activations and inhibitions in the growth of coleoptile and root system. The results showed that although the growth of the coleoptile was modified the root system was more susceptible to laser radiation, showing a maximum activation of $15.2 \%$ and a maximum inhibition of $17 \%$.
\end{abstract}

Keywords: laser diode, infrared laser radiation, wheat, coleoptile, root system 


\section{INTRODUCCIÓN}

El trigo es el segundo grano más producido a nivel mundial con una producción, reportada por la Organización de las Naciones Unidas para la Alimentación y la Agricultura (FAO), de 682 millones de toneladas métricas (Mt). En México desde el año de 1985 se ha observado una disminución paulatina en la producción de este grano (Villaseñor et al., 2007). Entre el año de 1996 y el año 2004 la importación de trigo pasó de 750 mil Mt a 3 millones de Mt de las 6 millones de Mt necesarias para satisfacer el consumo interno. En la actualidad la importación de trigo representa el $74.3 \%$ del total necesario para satisfacer la demanda interna.

En México existen dos ciclos de cultivo del trigo (Secretaria de Agricultura, Ganadería, Desarrollo Rural, Pesca y Alimentación, México, SAGARPA). El ciclo Otoño-Invierno, en el cual se produce el $90 \%$ de la producción interna, y el ciclo Primavera-Verano, en el cual se produce el 10\% restante de la producción interna. El ciclo de cultivo Primavera-Verano se ha considerado un ciclo de oportunidad para incrementar la producción anual de este grano en el país. Durante este ciclo de cultivo las semillas se siembran en un clima templado húmedo con alturas de entre los 2000 y 2800 metros sobre el nivel del mar, Tomando en consideración lo anterior es necesario buscar métodos que permitan mejorar el desarrollo de las semillas bajo estas condiciones para poder incrementar la producción en este ciclo de cultivo.

Una alternativa a los métodos químicos para el tratamiento de las semillas es el uso de métodos físicos, los cuales presentan la ventaja de no ser invasivos y tóxicos para las semillas (Aladjadjiyan, 2007; Vasilevski, 2003). El láser, desde su descubrimiento en 1960, ha sido considerado por los investigadores como un posible método físico de tratamiento de las semillas que permite mejorar el rendimiento, desarrollo y crecimiento de las plantas (Aladjadjiyan, 2007; Vasilevski, 2003; Dinoev et al., 2004; Podleśny et al., 2004, 2002; Rybiński et al., 2004; Salyaev et al., 2007, 2001). Los efectos reportados por los investigadores en diversas semillas de vegetales y cereales son la activación del desarrollo en etapas tempranas, aceleración e incremento de la germinación, modificación en la acumulación de la materia seca, incremento de la altura, peso y rendimiento de las plantas entre otros. Los parámetros de la luz láser que determinan la estimulación de las semillas son la longitud de onda, la densidad de potencia y el tiempo de exposición a la radiación (Aladjadjiyan, 2007; Dinoev et al., 2004). Otro parámetro que es de importancia pero que no corresponde a la radiación láser es la especie y variedad de las semillas.

Para la estimulación de semillas de trigo los láseres que más se han utilizado con este fin son los láseres de $\mathrm{He}-\mathrm{Ne}$ (con longitud de onda de 632.8nm), los láseres de diodos (con longitud de onda de 670nm) y los láseres de $\mathrm{CO}_{2}$ (con longitud de onda de $10600 \mathrm{~nm}$ ) (Drozd et al., 1998, 1999; Salyaev et al., 2007, 2001; Szajsner, 2003; Zong-Bo et al., 2008). Los dos primeros emiten luz en el espectro rojo (espectro visible) mientras que el tercero emite luz en el espectro infrarrojo (IR) lejano. Los efectos obtenidos en las semillas de trigo debido a su tratamiento con los láseres de He-Ne y los diodos láser han sido un mejoramiento en el crecimiento de las plántulas, un incremento en la germinación, en la longitud del tallo y de las raíces, así como en la densidad y en la longitud de las orejas de trigo. La radiación láser roja también ha permitido proteger las semillas contra el daño que produce la radiación UV-B. La radiación láser IR ha sido utilizada para mejorar la resistencia a la sequía de las semillas de trigo así como para mejorar algunos de sus parámetros de crecimiento. Los fotones producidos por los láseres IR tienen la característica de que penetran más en los tejidos pese a que su energía es menor que la producida por los láseres rojos.

Debido a la escasa información experimental acerca de los efectos de estimulación en semillas de trigo provocados por la radiación láser en el espectro IR cercano, el presente trabajo tiene como objetivo principal el investigar si este tipo de radiación, producida por un láser de diodo con longitud de onda de $980 \mathrm{~nm}$, estimula el crecimiento del trigo variedad Náhuatl (Triticum aestivum $L$ ) en sus etapas iniciales de desarrollo de forma tal que se aporte evidencia en favor del uso de este método físico como alternativa a los métodos químicos utilizados actualmente para incrementar la producción en el país en este ciclo de siembra. 


\section{MATERIALES Y MÉTODOS}

\section{Selección de la variedad de Trigo}

La variedad de trigo (Triticum aestivum $L$ ) seleccionada fue la Náhuatl F2000. Esta semilla fue proporcionada por el Instituto de Investigaciones Forestales, Agrícolas y Pecuarias (INIFAP, México) del país. Su desarrollo estuvo a cargo del instituto antes mencionado y del Centro Internacional de Mejora de Maíz y Trigo (CIMMYT, México). Esta variedad de trigo es utilizada en el ciclo de cultivo Primavera-Verano y es de importancia por su capacidad de desarrollo en los distintos estados de la república.

\section{Tratamiento con el láser IR}

La potencia de salida del láser de diodo con $\lambda=980 \mathrm{~nm}$ fue medida con un radiómetro piroeléctrico calibrado eléctricamente modelo Rs5900 conectado a una punta piroeléctrica de potencia modelo RjP735 de la compañía Laser Probe, USA. El patrón de radiación del láser fue medido con un sistema de fotodiodo diseñado en el laboratorio. Debido a que la radiación del láser de diodo no es uniforme, sólo el área central con disminuciones de potencia del $10 \%$ con respecto a la potencia máxima fue considerada para el tratamiento de las semillas.

Los parámetros del láser de diodo considerados para definir los tratamientos láser a aplicarse a las semillas de trigo fueron la densidad de potencia $\left[\mathrm{mW} / \mathrm{cm}^{2}\right]$ y el tiempo de exposición a la radiación láser [s]. Cada tratamiento $(\mathrm{T})$ se aplicó a un grupo diferente de semillas de trigo. Los tratamientos láser definidos fueron los siguientes: i) Exposición a una densidad de potencia de 15 $\mathrm{mW} / \mathrm{cm}^{2}$ con 5 tiempos diferentes: 15s (T1), 30s (T2), 60s (T3), 120s (T4), 240s (T5); ii) Exposición a una densidad de potencia de $30 \mathrm{~mW} / \mathrm{cm}^{2}$ con 5 tiempos diferentes: $15 \mathrm{~s}$ (T6), 30s (T7), 60s (T8), 120s (T9), 240s (T10); y iii) Exposición a una densidad de potencia de $60 \mathrm{~mW} / \mathrm{cm}^{2}$ con 5 tiempos diferentes: 15s (T11), 30s (T12), 60s (T13), 120s (T14), 240s (T15).

\section{Pruebas de crecimiento}

Los parámetros del crecimiento a evaluar en el trigo fueron la longitud del coleoptilo y la longitud del sistema radicular. Las pruebas de crecimiento fueron desarrolladas bajo condiciones de laboratorio de acuerdo con los lineamientos establecidos por la Asociación Internacional de Pruebas de Semillas (ISTA 2009 y 2010). Para cada tratamiento láser se consideraron 16 repeticiones $(n=16)$, con 25 semillas cada una, dando un total de 400 semillas a tratar. Cada densidad de potencia presentó un control con la misma cantidad de semillas. Después de haber tratado las semillas con la radiación láser, estas fueron colocadas sobre papel filtro y enrolladas. Posteriormente el papel filtro, con las semillas, fue humedecido con la misma cantidad de agua destilada. Para las semillas de control se aplicó el mismo procedimiento. Los papeles filtro con las semillas tratadas y los controles fueron marcados e introducidos de forma aleatoria en una cámara de germinación por 10 días. La temperatura y humedad para las pruebas de crecimiento dentro de la cámara fueron de $20 \pm 2^{\circ} \mathrm{C}$ y de $70 \pm 2 \%$ respectivamente.

\section{Análisis estadístico}

Después del décimo día se procedió a medir la longitud del coleoptilo y del sistema radicular. Una vez registrados los valores de crecimiento de ambas variables se procedió a analizar la normalidad de los datos a través de la prueba de Kolmogorov-Smirnov. Posteriormente los datos fueron sometidos a un análisis de varianza (ANOVA) para detectar diferencias entre los parámetros de las medias. Estas a su vez fueron comparadas utilizando la prueba de Tukey (múltiples comparaciones) para detectar diferencias entre el control y las semillas tratadas.

\section{RESULTADOS Y DISCUSIÓN}

Aladjadjiyan (2007) y Vasilevski (2003) mencionan que la radiación láser permite a las semillas vegetar con un nivel mayor de energía, lo que trae como consecuencia la estimulación del 
crecimiento en las etapas iniciales de su desarrollo. En la presente investigación se observó que para una longitud de onda constante $(\lambda=980 \mathrm{~nm})$, los cambios en la densidad de potencia y el tiempo de exposición permiten no solo incrementar el crecimiento de las semillas de trigo sino también inhibirlo. Tanto la longitud del coleoptilo como la del sistema radicular mostraron incrementos y decrementos importantes en su desarrollo en función de la densidad de potencia y del tiempo de exposición utilizado.

\section{Crecimiento del coleoptilo}

De todos los tratamientos aplicados solo 3 de ellos produjeron cambios estadísticamente significativos $(\rho)$ en el crecimiento del coleoptilo: dos de los tratamientos láser, T3 y T4 con $\rho<$ 0.001 , produjeron una reducción importante en la longitud del coleoptilo, $11 \%$ y $12 \%$ respectivamente (Fig. 1); el tercer tratamiento, T13 con $\rho<0.01$, incrementó la longitud del coleoptilo $6.5 \%$ (Fig. 2). Los tratamientos T3 y T4 presentaron una densidad de potencia de $15 \mathrm{~mW} / \mathrm{cm}^{2}$ y tiempos de exposición de $60 \mathrm{~s}$ y 120 s respectivamente, mientras que el tratamiento T13 presentó una densidad de potencia de $60 \mathrm{~mW} / \mathrm{cm}^{2}$ y un tiempo de exposición de $60 \mathrm{~s}$.

En función de la significancia estadística y de los porcentajes de crecimiento obtenidos es posible concluir que este tipo de radiación láser produce efectos de inhibición de mayor amplitud en comparación con los efectos de activación para el crecimiento del coleoptilo.

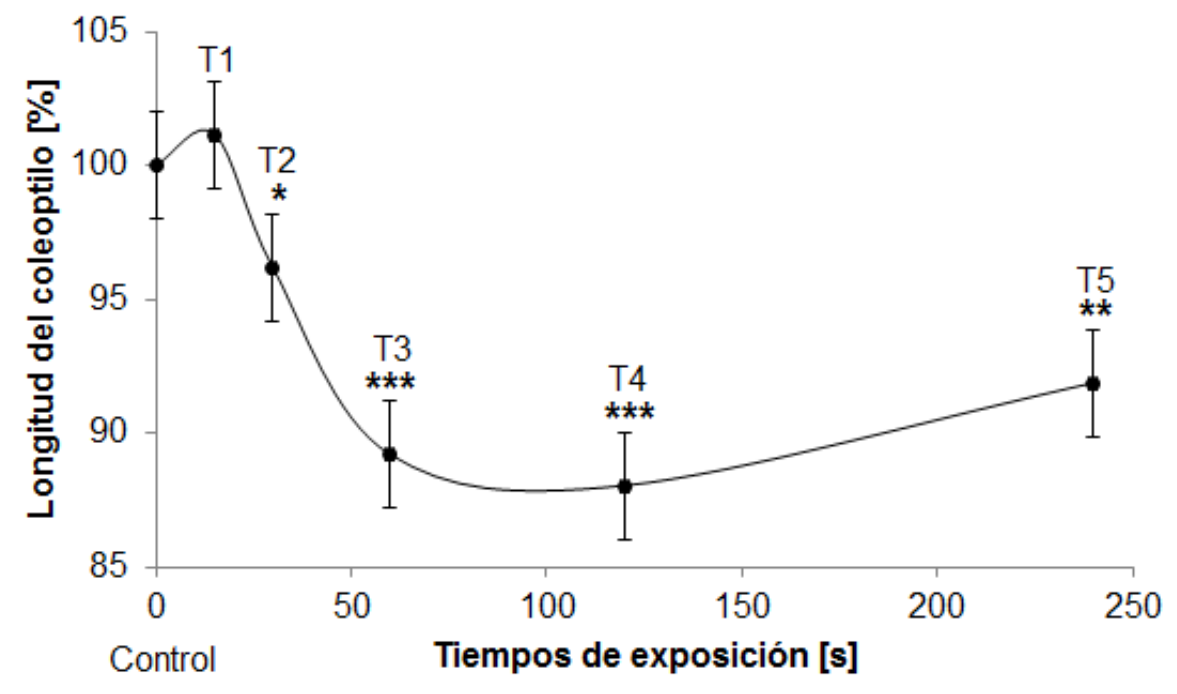

Fig. 1: Crecimiento del coleoptilo para una densidad de potencia de $15 \mathrm{~mW} / \mathrm{cm}^{2}$.

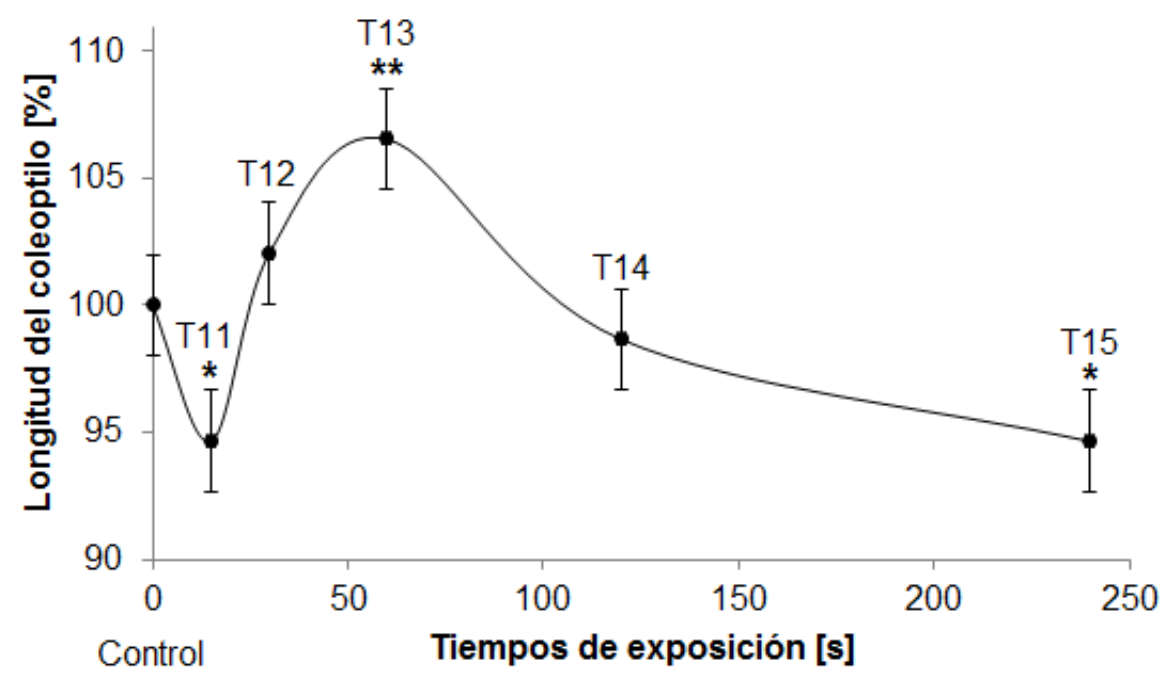

Fig. 2: Crecimiento del coleoptilo para una densidad de potencia de $60 \mathrm{~mW} / \mathrm{cm}^{2}$. 


\section{Crecimiento del sistema radicular}

La longitud del sistema radicular registró dos crecimientos máximos y dos inhibiciones máximas con respecto al control los cuales fueron estadísticamente significativos $(\rho<0.001)$ y comparables en magnitud. Los dos crecimientos máximos se obtuvieron para una densidad de potencia de $60 \mathrm{~mW} / \mathrm{cm}^{2}$ y tiempos de exposición de 30s (T12) y 60s (T13): las magnitudes fueron del $15.2 \%$ y $13.6 \%$ respectivamente (Fig. 4). Las dos inhibiciones máximas en el crecimiento del sistema radicular fueron obtenidas para una densidad de potencia de $15 \mathrm{~mW} / \mathrm{cm}^{2}$ y tiempos de exposición de 120s (T4) y 240s (T5): las magnitudes de estas inhibiciones fueron del $17 \%$ y $13 \%$ respectivamente.

De los resultados obtenidos para el crecimiento del coleoptilo y el sistema radicular es posible concluir que este último fue más susceptible a los tratamientos láser que el primero mostrando de esta forma activaciones e inhibiciones mayores.

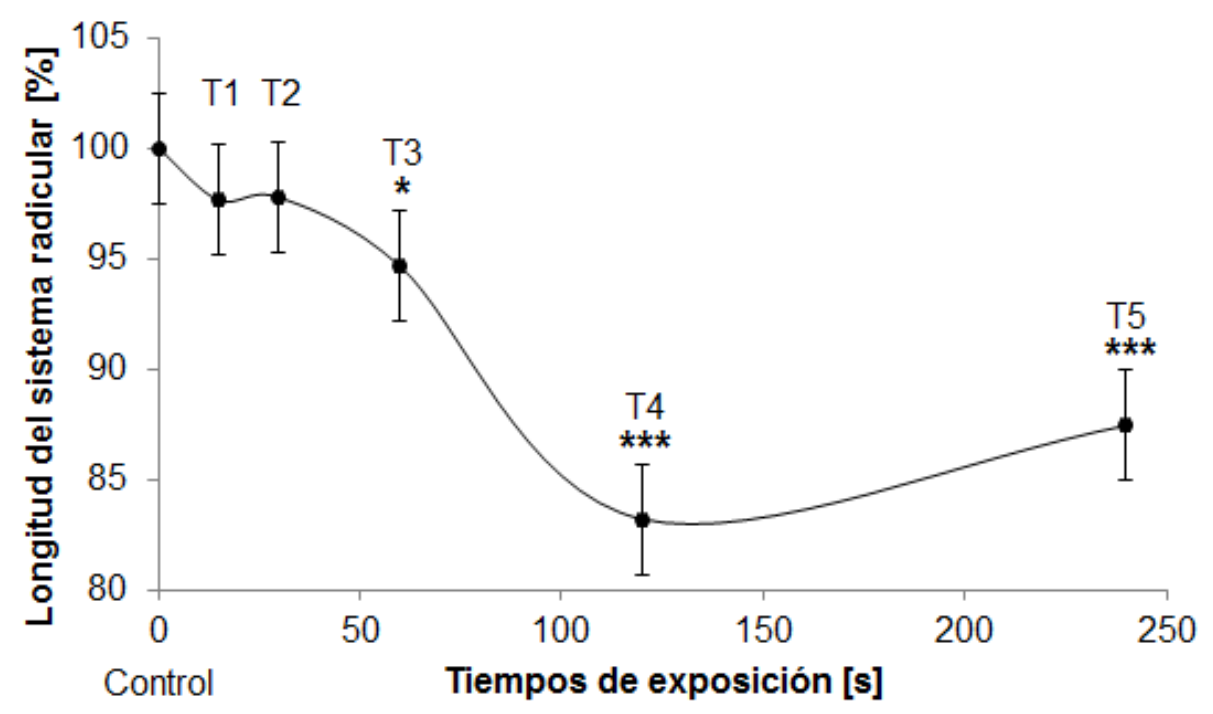

Fig. 3: Crecimiento del sistema radicular para una densidad de potencia de $15 \mathrm{~mW} / \mathrm{cm}^{2}$.

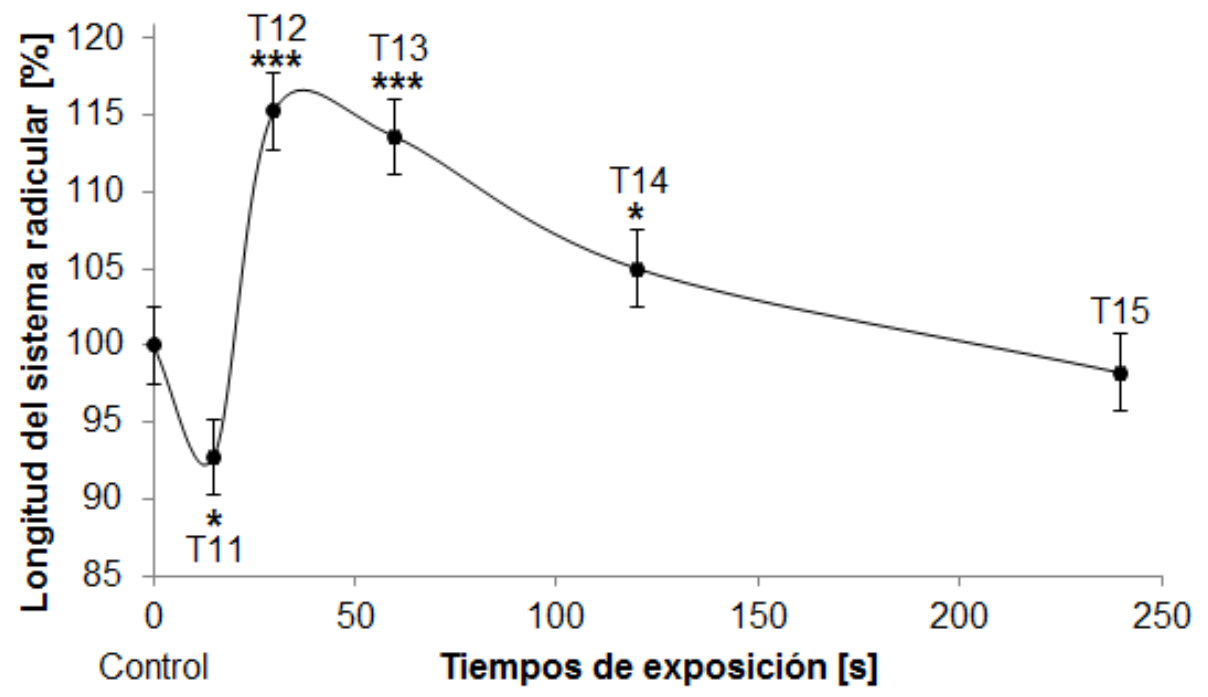

Fig. 4: Crecimiento del sistema radicular para una densidad de potencia de $60 \mathrm{~mW} / \mathrm{cm}^{2}$. 


\section{CONCLUSIONES}

La radiación láser IR con longitud de onda $\lambda=980 \mathrm{~nm}$ producida por un láser de diodo permite activar o inhibir el crecimiento del coleoptilo y del sistema radicular de la semillas de trigo en función de la densidad de potencia y del tiempo de exposición seleccionado para su tratamiento antes de la siembra.

El efecto de mayor amplitud obtenido en el crecimiento del coleoptilo fue de $12 \%$ por debajo del nivel de control (efecto inhibitorio del crecimiento) para una densidad de potencia de $15 \mathrm{~mW} / \mathrm{cm}^{2}$ y tiempo de exposición de 120s (T4).

El sistema radicular fue el más sensible a la radiación láser IR mostrando diversas activaciones e inhibiciones en su crecimiento. La máxima activación fue del $15.2 \%$ por arriba del nivel de control para una densidad de potencia de $60 \mathrm{~mW} / \mathrm{cm}^{2}$ y tiempo de exposición de $30 \mathrm{~s}$ (T12), mientras que la máxima inhibición fue del $17 \%$ por debajo del nivel de control para una densidad de potencia de $15 \mathrm{~mW} / \mathrm{cm}^{2}$ y tiempo de exposición de $120 \mathrm{~s}$ (T4).

No todos los tratamientos láser $(T)$ produjeron cambios en el crecimiento del coleoptilo y del sistema radicular. Para una longitud de onda constante la densidad de potencia y el tiempo de exposición a la radiación láser determinan el efecto así como la amplitud del mismo.

\section{REFERENCIAS}

Aladjadjiyan Anna, The use of physical methods for plant growing stimulation, J. Cent. Eur. Agric., 8(3), 369-380 (2007).

Dinoev St., Antonov M., Stoyanov T. y Georgieva Chr. Spectral impact of low-power laser radiation on wheat and maize parameters, Bulgarian Academy of Sciences, 54(1), 74-85 (2004).

Drozd D. y Szajsner H., Influence of presowing laser radiation on spring wheat characters, Int. Agrophysics, 13(1), 79-85 (1999).

Drozd D. y Szajsner H., Effect of laser radiation on spring wheat genotypes, Int. Agrophysics, 13(2), 197-202 (1998).

Podleśny J. y Podleśna, A., Morphological changes and yield of selected species of leguminous plants under the influence of seed treatment with laser light, Int. Agrophys., 18(3), 253-260 (2004).

Podleśny J., Effect of laser irradiation on the biochemical changes in seeds and the accumulation of dry matter in the faba bean, Int. Agrophys., 16(1), 209-213 (2002).

Rybiński W. y Garczyński S., Influence of laser light on leaf area and parameters of photosynthetic activity in DH lines of spring barley (Hordeum vulgare L.), Int. Agrophys., 18(3), 261-267 (2004).

Salyaev R. K., Dudareva, L. V., Lankevich, S. V., Makarenko S. P., Sumtsova, V. M. y Rudikovskaya E. G., The effect of Low-intensity laser irradiation on the chemical composition and structure of lipids in wheat tissue culture, Dokl. Akad. Nauk., 412, 87-88 (2007).

Salyaev R. K., Dudareva, L. V., Lankevich, S. V. y Sumtsova, V. M., The effect of Low-intensity coherent radiation on morphogenetic processes in wheat callus culture, Dokl. Akad. Nauk., 376, 113-114 (2001).

Szajsner Hanna, Comparision of the spring and winter wheat forms reactions to the pre-sowing laser biostimulation, Acta Agrophysica, 2(3), 639-643 (2003).

Vasilevski G., Perspectives of the application of biophysical methods in sustainable agriculture, Bulg. J. Plant Physiol., 29(3), 179-186 (2003).

Villaseñor Mir H. E., Espitia Rangel E., Huerta Espino J., Solís Moya E., González Iñiguez R. M., Osorio Alcalá L. y Pérez Herrera P., Triunfo F2004, new bread wheat cultivar for rainfed conditions in Mexico, Agricultura Técnica en México, 33(3), 319-322 (2007).

Zong-Bo Qiu, Xiao Liu, Xiang-Jun Tian y Ming Yue, Effects of CO2 laser pretreatment on drought stress resistance in wheat, Journal of Photochemistry and Photobiology, 90(1), 17-25 (2008). 